\title{
Toward health equities, social justice, and emancipatory calls for knowledge development in nursing
}

\author{
Areej Al-Hamad* \\ School of Nursing, University of Calgary in Qatar, Qatar
}

Received: March 29, 2020

DOI: $10.5430 /$ jnep.v10n8p66

\author{
Accepted: May 7, 2020 \\ Online Published: May 18, 2020 \\ URL: https://doi.org/10.5430/jnep.v10n8p66
}

\begin{abstract}
Knowledge development within the nursing discipline requires an exploration of ethical, philosophical, and spiritual contexts to make nursing a unique discipline within a health-care system. Intersectionality theory as a philosophical paradigm has a potential contribution to the nursing discipline and public health. Moreover, intersectionality is inevitably intertwined with various dimensions of knowledge development in nursing where gender and culture are therefore seen as large categories of knowledge construction. Adopting an intersectional approach that encompasses health equity, social justice, and emancipatory perspectives helps the nurse to gain a better understanding of the life contexts and experiences of the members of a marginalized group, which ultimately helps to enhance their health and quality of life. Therefore, it appears axiomatic that an intersectional approach could yield fruitful insights into public health and knowledge development in nursing.
\end{abstract}

Key Words: Intersectionality, Knowledge development, Nursing, Public health, Health equities, Social justice.

\section{INTRODUCTION}

"If we aren't intersectional, some of us, the most vulnerable, are going to fall through the cracks". ${ }^{[1]}$ Intersectionality is an emerging philosophical, research, and policy paradigm in the nursing discipline that is gathering support due to its unique potential for defining and interpreting nursing phenomena. ${ }^{[2-10]}$ Davis $^{[11]}$ stresses that the growing interest in using this paradigm is due to its vagueness and openendedness, features which attract renowned feminist scholars in nursing and other disciplines and of various political persuasions to apply it in their research. The main reason for the adoption of intersectionality in nursing may be its relevant, distinct tenets that epitomize both its uniqueness and potential as a paradigm. These tenets are fivefold. First, human lives cannot be viewed from a single perspective. Second, human experiences cannot be grasped or comprehended by focusing on one single factor. Third, social categories, positions, and identities such as gender, culture, class, race, and immigration, are interrelated, dynamic, and socially constructed. Fourth, social locations are interconnected and shaped by the intersection of social processes and structures that are influenced by a power differential. And last but not least, the call for social justice, emancipation from oppression, and equity for historically marginalized groups. ${ }^{[7,8,12]}$

However, the varying degrees of success in utilizing intersectionality as a philosophical framework in the nursing profession has meant that its application in public health nursing has remained underdeveloped. ${ }^{[13]}$ Nevertheless, there seems

*Correspondence: Areej Al-Hamad; Email: areej.alhamad@ucalgary.ca; Address: School of Nursing, University of Calgary in Qatar, Qatar. 
to be significant merit in adopting an intersectional framework in public health nursing and in continuing to strengthen research in this area. Thus, my intention in this paper is to enhance awareness about the potential of intersectionality as a philosophical framework in the field of public health nursing and also to highlight the relative helpfulness of the mutually constitutive nature of intersectionality in nursing research, practice, and policy. A further aim is to provide a theoretical justification as well as a contextual explanation for how intersectionality shapes and informs public health nursing.

The remainder of this paper is organized as follows: First, I explore my philosophical position, my epistemological and ontological precepts, and my various ways of knowing, and how these are connected and well-matched to the tenets and assumptions of intersectionality. Next, to move the debate forward and to strengthen the argument in support of adopt ing the intersectionality paradigm, I consider the possible merits of incorporating intersectionality into public health nursing research, practice, and policy. Then, I discuss some of the ways in which incorporating intersectionality in public health nursing could contribute to knowledge development and advance the nursing discipline.

\section{THE AUTHOR'S ONTOLOGICAL, EPISTE- MOLOGICAL, AND PHILOSOPHICAL POSI- TION}

In this section, I explore my epistemological and ontological questioning according to the patterns of knowing described by Carper (1978). I would argue that my philosophical position, which has evolved through my ontological and epistemological reflections on ways of knowing, helps me to respond to and critique any foundational changes in the nursing discipline and to make a substantial contribution to the bases of nursing. I strongly believe that a discussion of my epistemological knowledge and my ontological perceptions of reality through various types of questioning helps me to understand how, where, when and why I practice nursing.

The primary reason for my interest in applying intersectionality in public health nursing relates to its relevance to the five principles of public health care, namely, accessibility, public participation, appropriate technology, wellness promotion and illness prevention, and intersectoral collaboration. ${ }^{[4]}$ My interest was ignited by my personal experience as an immigrant nurse and fostered by my curiosity, my conscious immersion in a new culture, and my scientific motivation that has developed over the years through academic discourse on, working with, and studying marginalized populations including homeless and refugee women. Indeed, intersec-

Published by Sciedu Press tionality is well-aligned with my ontological outlook as a public health nurse because I constantly strive to call for health equity and social justice in my nursing care. As a public health nurse, my nursing practice involves trying to provide fair and just care as well as advocating for the interest of marginalized population, and this has helped to shape my understanding of the different narratives and voices of marginalized communities. Also, as a public health nurse, I attempt to engage in a philosophical inquiry and theoretical exploration to reveal the relevance and the applicability of intersectionality in public health nursing.

The key question that has driven me as a nurse, researcher, and educator to examine the systemic and structural marginalization of Syrian refugee women in health-care interactions and settings is: How do inequality, injustice, and oppression affect the health outcomes of a marginalized population? In seeking to answer this question, I feel that the concept of "holistic care" resonates with me in my capacity as a public health nurse because the health of Syrian refugee women is affected by every aspect of their life, including food, housing, education, violence, migration, and frequent displacement. Indeed, from the standpoint of my own philosophical position, I was intrigued by Crenshaw's ${ }^{[5]}$ assertion that there is a need to employ a unique theoretical lens to address various forms of marginalization that is geared toward the complexities of social identities in relation to micro and macro-level social structures. This philosophical perspective has clearly influenced the field of nursing, as evidenced by the work of various authors. ${ }^{[3,12,14-17]}$

Epistemologically, I have asked various questions related to knowledge development in nursing. The reflections on these questions make substantial philosophical contributions to the theoretical foundations of my nursing practice that should not be neglected. Furthermore, these questions help me to understand how various identities with all their richness and diversity have converged into a variety of patterns of knowing that may move the nursing profession away from the medical and other scientific disciplines. Moreover, from an ontological perspective, I have started to ask questions regarding the nature and meaning of my own and my clients' realities and being. I would say that this ontological questioning differs from the epistemological questioning of the scope and justification of knowledge. Arguably, this difference in questioning has been conceptualized by various scholars in the field of nursing as a philosophical expansion from the epistemological to the ontological. ${ }^{[18-21]}$

From a research perspective, my studies are usually informed by listening to patients and to marginalized individuals because I believe that it is the patients or the individuals 
themselves who are the experts in their own well-being. In addition, from my experience of researching marginalized women, including homeless women and Syrian refugee women, I have realized that "being heard" or giving marginalized women their voice is what these women want most from the nursing scholar. Moreover, adopting an intersectional approach that encompasses health equity, social justice, and emancipatory perspectives helps the nurse to gain a better understanding of the life contexts and experiences of the members of a marginalized group, which ultimately helps to enhance their health and quality of life. ${ }^{[14]}$ Lastly, because I am interested in the social determinants of health, it appears axiomatic that an intersectional approach could yield fruitful insights into public health nursing.

Generally, the epistemological assumptions of intersectionality lead to more relevant and organic understandings of various ways of knowing in nursing. ${ }^{[22-24]} \mathrm{Carper}^{[18]}$ asserts that nurses' multiple patterns of knowing include the empiric, esthetic, personal and ethical. Personally, I can see myself delving into and wisely using all these patterns of knowing without rejecting or neglecting any of them. A consideration of all the patterns of knowing assists me in understanding the dynamics and congruence between the patterns and their goals. ${ }^{[18]}$ Indeed, I was intrigued by Carper's view that the four patterns of knowing are ever emerging rather than static. Carper's viewpoint helps me to recognize that my knowing and my practice are complex, diverse, and ever emerging and it also informs the way in which I structure my nursing care and profession. For instance, adopting Carper's view assists me to expand my understanding and way of thinking about the various types of knowledge and theory needed in my practice as public health care provider working within discipline such as nursing.

From the perspective of the empiric pattern of knowing, an epistemological question could be: How do I come to know the knowable? Carper (1978) suggests that one approach to answering this question is to focus on the end products of knowing rather than on the process of how we come to know. Arguably, I can relate this epistemological question to my own epistemological area of interest, that of using intersectionality in nursing and particularly public health nursing, by focusing on women's empowerment, social justice, and emancipation from oppression as outcomes of adopting intersectionality rather than placing the emphasis on the ways and means of achieving these outcomes. For me, this argument explains the interrelationships between my epistemological and ontological awareness and understanding of the empiric world and my relation to it. Therefore, I could suppose that my empirics-driven epistemological question might evolve into an ontological question if I were able to understand the world around me by grasping the relationship between what I know about intersectionality and the marginalized group and how the things that I know are related. Thus, my empiricsbased ontological question could be: How do I find meaning in what I know about intersectionality and its assumptions, intentions, and influence on public health and in dealing with a marginalized group within the nursing discipline? The interrelatedness between what I know through my multiple patterns of knowing and various experiences related to the issue of social justice that I have encountered in my practice helps me to be an advocate for the marginalized group.

In describing the esthetic pattern of knowing, Carper ${ }^{[18]}$ asserts that it relates to the art of nursing and she emphasizes the importance of empathy, proportion, and unity as they relate to the whole. One possible epistemological question in this regard would be: How do I come to know the art of nursing? Within this notion of esthetics, a proposed ontological question could be formulated as: What does my perception of the art of nursing reveal to me? This ontological question reflects the significance of perception in the relation between empathy and esthetics in nursing. ${ }^{[18,25]}$ Thus, I can gain knowledge of an individual's experience (e.g. a Syrian refugee woman's experiences) through my empathic knowing. This ontological questioning thus suggests that multiple realities can possibly be revealed to us through our perceptions and empathizing with others [18]. To relate this way of knowing to intersectionality, it is clearly shown that practicing empathizing with historically oppressed and marginalized groups can improve our understanding of the meaning and the reality of various social and power structures in a particular society. Thus, esthetic knowing illustrates the art of nursing and how the art of public health nursing and the effect of perceptions and empathy can inform and provide meaning to the reality of knowing and being. ${ }^{[18,25]}$

Personal knowledge is another important pattern of knowing in nursing, and for me it is an essential pattern for understanding the meaning of health and the well-being of the individual. ${ }^{[18,25]}$ One possible epistemological question that might arise in relation to this pattern of knowing might be: How do I come to know who I am as a nurse? In fact, this epistemological question implies that the focus is not about "knowing about the self," but about "knowing the self" ${ }^{[18,25]}$ Thus, the main assumption of this pattern of knowing echoes my ontological question: Who am I? This ontological question compels me to return to my beginnings as an immigrant public health nurse who has experienced some degree of cultural marginality. In other words, my personal meaning and multiple realities that may emerge from my personal knowing of different forms of oppression and marginalization are embodied in the narratives of the vulnerable population. 
Therefore, my unique intersectional personal narratives, as well as the marginalized individuals' narratives or stories, reflect our existence.

As for the ethical pattern of knowing, the ethical component of nursing emphasizes what ought to be done. ${ }^{[18,25]}$ Thus, an epistemological question could be: How do I come to know what I morally ought to do? This ethical pattern of knowing resonates with me and my interests because I postulate that my ontological focus is: Who morally ought I to be? This differs from my epistemological focus on the knowledge needed to determine and judge the moral values of what I ought to do based on my various intentions, motives and character traits. ${ }^{[18,25]}$ Thus, this complexity and richness of ethical knowing reflects the unique nature of intersectionality in capturing the multidimensional components of ethics in nursing. Moreover, in current contemporary nursing practice, this pattern of knowing bears some resemblance to the nurses' code of ethics and the intention of intersectionality to achieve social justice and health equity.

My other way of knowing is emancipatory knowing, a type of knowing proposed by Chinn and Kramer ${ }^{[22]}$ where they call on nurses to develop their own emancipatory knowing so the invisible becomes visible and the voiceless becomes voiced through the process of enlightenment and the sociopolitical forces upon marginalized individuals. My outcomes of emancipatory knowing including a recognition of the impact of injustice or inequity, to inform change by considering complex aspects of experience and context to change a situation to the one that improves people's lives. These outcomes of emancipatory knowing help me to better understand how hegemony in nursing might perpetuate oppression and domination.

\section{WHAT DOES INTERSECTIONALITY HAVE TO OFFER TO NURSING RESEARCH, PRAC- TICE, AND POLICY?}

\subsection{Research}

Intersectionality has been the subject of a great deal of academic interest because it offers a unique and relevant conceptual pespective from which to study marginalized populations. ${ }^{[6,8,12,13,15,24,26]}$ Particularly, intersectionality has the potential to enrich public health research through focusing greater attention on the pathways and processes involved in producing health inequalities. For instance, Kapilashrami, Hill $^{[13]}$ argue that an intersectional framework is relevant and applicable to research on social justice and emancipatory and health inequalities because it obliges nurse researchers to move beyond gender, race, class and socioeconomic position in analyzing the structural determinants of health. Specifi- cally, I would argue that an intersectional account of social position and identity stimulates nurse researchers to focus on social determinants of health such as food, housing, income and education rather than social categories, and to approach the experiences of relative disadvantage as one form of power differential across the social organization.

Engaging in an intersectional approach allows nurse researchers to address the multiple categories of identity and social systems and how their intersection can relate to inequality where the relationship between the categories is open and equal, and all the relevant categories are mutually constituted to each other. ${ }^{[3,7,13,27,28]}$ The intersectional approach enables nurse researchers to critically examine the simultaneous impact of resistance to systems and various structures of oppression and domination on health equity and social justice within the nursing discipline.

On the other hand, the intersectionality theory is somewhat circumspect regarding how researchers, public health practitioners, and policymakers perceive social categories as well as about the complex relationship between various constituting factors of social location and structures of oppression. ${ }^{[3,4,6]}$ Methodologically, the theoretical assumptions of intersectionality support the use of an interdisciplinary approach to achieve the key goal of empowerment. ${ }^{[2-4,6,8,12]}$ However, one emerging issue within the field of intersectionality-driven nursing research is developing methods that can effectively translate intersectionality theory into practical guidelines that can be utilized by researchers, decision makers, and policymakers. ${ }^{[6-8]}$ In the same vein, Bowleg ${ }^{[4]}$ argues that conducting an intersectionality analysis can be extremely intimidating due to the limited methodological choices available, which calls for a methodological revolution to facilitate the application of intersectionality to public health policies and research.

Based on my understanding as a public health nurse and researcher of the relationship between social position and health inequities, I think that nurse researchers need to be cognizant of the commonalities of and differences between the various drivers and causes of health inequalities in their analyses of systems of oppression, domination, or discrimination and power relations. For instance, in Canada Syrian refugee women may have a unique life experience, and access to health-related resources, that differs to Syrian refugee women living in other countries. Thus, placing these women in the same category as Syrian refugee women elsewhere makes it challenging to understand how various aspects of social location may affect these women's lives. Therefore, I believe that intersectionality has a unique potential for addressing the health-care needs and health equity of marginal- 
ized populations including Syrian refugee women, and the results of its application to this issue will contribute to our understanding of and provide reasonable grounds for the use of an intersectionality framework in wider public health research.

\subsection{Practice}

As mentioned in the introduction, the intersection between gender, race, culture and refugee status as crucial categories, and forms of oppression serves to expand our scope of utilizing intersectionality beyond such categories to be included in our nursing practices. ${ }^{[3,4,8,14,19]}$ A point of departure worthy of a discussion here is the contribution of intersectionality to nursing practice. As health-care providers, I believe it is crucial that nurses use well-developed theories to inform their practice. This echoes ${ }^{[20]}$ discourse, where she posits that theory offers the basis to understand the reality of nursing and allows nurses to understand the rationale behind an event. Indeed, intersectionality-driven nursing care and practice takes into account the racial and cultural stereotypes that may affect or interfere with the delivery of care to patients from diverse racial, ethnic, and cultural backgrounds. Moreover, intersectionality-based practices allow nursing professionals to explore the individual experiences of various immigrants, refugees, and marginalized communities. ${ }^{[17,23,29]}$ The exploration of such experiences fosters mutual respect, tolerance, and nonjudgmental acceptance among nurses and might mitigate the issue of racism in nursing. As a public health nurse, I strongly believe that being able to nonjudgmentally understand, accommodate, and appreciate each individual's unique background has a strong influence on how the illness is perceived by the patients themselves and by the healthcare providers. Furthermore, it is essential to uphold the concept of social justice and health equity as a universal imperative at all times when dealing with any marginalized population despite pressures that include various forms of social, political, and budgetary constraints. ${ }^{[29]}$ In fact, calling for social justice and empowerment and advocating emancipatory nursing care are aligned as mutually constitutive and intersectionality researchers have confirmed their symbiotic existence. ${ }^{[2]}$ In fact, intersectionality offers a shift from the traditional enclave of culturally marginalized groups to the wider perspective of dialectic discussion and agenda to achieve social justice and empowerment. ${ }^{[8,13,14]}$ Generally, intersectionality-guided nursing practice allows nurses to move beyond a unidimensional focus on social class, identity or position to consider and address the multiple systems of privilege and oppression that are of relevance to the health of the marginalized group. ${ }^{[8,12,13,30]}$ Furthermore, this type of nursing practice brings to the fore the health disparities and the oppression of marginalized groups and reveals how multiple systems of privilege and oppression (such as race, culture, refugee and immigration) intersect within the various social-structural levels to exacerbate health inequalities. Also, addressing the macro social-structural factors offers us a chance to understand how social systems and resources could maintain or even reinforce the health inequalities of a marginalized population. Such understandings may inform the development of empowerment-based interventions and practices to unpack the processes and structures through which multiple social positions and inequalities may reinforce health inequalities of marginalized groups. Moreover, the intersectionality approach contributes to public health by describing the result of adverse health outcomes at the intersection of high- and low-status identities. ${ }^{[8]}$

In this regard, I would say that adopting an intersectional stance has affected my practice in two ways. First, the approach takes into consideration the structural inequities that cause health issues and also explores the power differential that provides privilege to some individuals while marginalizing others. ${ }^{[19]}$ Second, this approach allows me to label my practice as emancipatory, where I emphasize health care challenges and the structural inequities that might affect the health of marginalized population and also advocate for social justice for vulnerable communities. Thus, intersectionality provides a more comprehensive insight into how various women's social identities intersect in complex ways to show social inequality and discrimination. The discussion to this point suggests that public health nurses and practitioners should advocate for a greater emphasis being placed on how social-structural factors beyond the level of the individual affect health. ${ }^{[8,31]}$ Nurses need to move beyond merely depicting the experience of the oppressed group and their health inequalities to explore the social processes and structures that reinforce their inequalities by examining the foundation of privilege and the power differential within society. ${ }^{[13]} \mathrm{Un}$ fortunately, the pervasiveness of and heavy reliance on new technologies has affected our contemporary nursing practices, particularly the value of cultural behaviors, practices, and meaning within the context of holistic, culturally safe, and individualized nursing care. For instance, adopting best practice guidelines that are informed by and recommended the use of intersectionality in health care could be an evidence of the potential of intersectionality to improve the quality of care and call for justices for the marginalized group. Generally, there are groups, including immigrants and refugees, that are reluctant to seek health care due to different cultural beliefs and practices. ${ }^{[32-34]}$ In order to address the needs of such groups, the nurse needs to be well prepared and have knowledge and awareness of cross-culture conflict and should also follow culturally safe practices for 
that group. Indeed, intersectionality welcomes the idea of cultural claiming, where culture is seen as a defense that women from minority cultures (e.g. Syrian refugee women) use against predominant cultures. ${ }^{[32-35]}$ In other words, the intersection of gender, race, and cultural oppression call for a commitment to emancipatory and intersectionality-guided nursing practices that permit marginalized cultural groups to protect their cultures and identities.

From the above delineation, intersectionality assumptions could be posited as an epochal practical guide for the nursing discipline and particularly for the public health domain with respect to caring for a culturally marginalized population. To summarize, I would contend that intersectionality is a paradigm that is both appropriate and harmonious with contemporary nursing practices in which cultural marginality is embedded. Thus, I would argue that intersectionality offers a comprehensive framework for nursing practice.

\subsection{Policy}

From a health policy perspective, intersectionality can also add depth to policy analysis. ${ }^{[7]}$ There has been a growing interest in intersectionality theory and its potential to promote social justice and equity-driven health policy analyses. ${ }^{[7,8,16,26]}$ The novelty of an intersectionality-based policy analysis lies in its inclusion of different layers to capture and respond to the multi-level interplay among the social locations, identities, forces, and power structures that shape and inform human life and health. ${ }^{[7,8]}$ Moreover, an intersectionality-based policy analysis allows policymakers to illuminate how individuals' and groups' power differentials and privileges, oppression, socioeconomic status, political status, health, and well-being may affect and inform policy constructs. ${ }^{[7,8]}$ Significantly, developing intersectionalitybased health policies can succinctly distill the value of social justice and health equity to capture the different dimensions of health, politics, the lived experiences of marginalized populations, knowledge, and intersecting social positions and identities. ${ }^{[7]}$ In addition, adopting an intersectional lens through which to examine nursing policies assists in generating transformative insights and policy solutions and actions that are driven by equity-focused policy frameworks. ${ }^{[7,8]}$ Collins $^{[?]}$ argues that intersectionality-based policy analysis needs to consider four domains of power: the structural domain, which encompasses laws and institutions where these methods are applied; the disciplinary domain, which includes administrative and bureaucratic management aspects; the hegemonic domain, which questions the cultural and ideological relationships of domination; and finally, the interpersonal domain, which covers and embraces individuals' everyday interactions that are influenced by their relation to

Published by Sciedu Press various forms and categories of inequity.

In the same vein, Yuval-Davis ${ }^{[37]}$ asserts that intersectionality-based policy analysis is a constitutive approach that takes into account the macro and micro dimensions of structural and power differential. More specifically, Yuval-Davis $^{[37]}$ proposes intersectional-based analytical framework, which consists of four levels. The first is an organizational analysis that relates to the organization or institution's social, political, and economic agenda. The second is an intersubjective analysis that addresses the power relations between individuals in particular situations. The third is an experiential analysis that touches on the subjective experience and the perception of individuals and their attitudes toward others. Finally, the fourth is a representational analysis that takes into accounts the cultural representations of various social structures and divisions within a particular society. In light of the above, it would seem that intersectionality theory has great potential in terms of its application in the field of public health policy.

\section{INTERSECTIONALITY AND KNOWLEDGE DEVELOPMENT IN NURSING}

Knowledge development within the nursing discipline requires an exploration of ethical, philosophical, and spiritual contexts, which in turn makes nursing a unique discipline within a health-care system. ${ }^{[21,22]}$ Indeed, intersectionality is inevitably intertwined with various dimensions of knowledge development in nursing where gender and culture are therefore seen as large categories of knowledge construction. ${ }^{[38]}$ Moreover, intersectional public health research may assist in both the development and testing of new nursing theories. ${ }^{[9,31,39,40]}$ Intersectionality theory also challenges the traditional reliance on one methodology or method to generate knowledge. Here, my core argument is that the adoption of an intersectionality perspective in the nursing profession may strengthen the robustness of the research findings on health inequalities, emancipatory nursing care, and social justice. To elaborate the significance and relevance of an intersectional perspective in the nursing discipline, I propose an approach that resonates with the holism principles of knowledge development, where various social locations, positions, and identities are perceived as more than the sum of their parts. Interestingly, this approach mirrors the underlying assumption of intersectionality in terms of the intersecting of multiple categories, social identities, and related systems of oppression, domination, or discrimination. Moreover, intersectionality seems to be a potentially fruitful framework for advancing the nursing discipline because it considers the effect of the interconnections of various social positions in the accomplishment of the various goals of nursing. 
From an intersectional perspective, Bilge ${ }^{[2]}$ argued that knowledge development could be achieved through questioning the potential of various axes, categories, power and social structures of health inequality and inequities within specific communities. Another potential implication of intersectionality theory in knowledge development is its novelty to addressing power differential between the advantaged and disadvantaged groups for the sake of health equity, empowerment, and social justice. ${ }^{[8,13,14,19,24]}$

\section{Conclusion}

In this paper, I explored intersectionality theory as a philosophical paradigm and highlighted its potential contribution to the nursing discipline in general and public health nursing in particular. Intersectionality theory can be applied to reveal and decipher the complexities of various power and social structures in order to gain a fuller understanding of the extent of health and social inequities. Intersectionality as a theory, like public health nursing, was developed in order to try to improve the public's health and to achieve health equity for everyone. The commitment to the goal of health equity in the practice of public health nursing means that the integration of intersectionality into public health nursing is an ideal marriage due to the shared aims and assumptions regarding the promotion of health equity and social justice for marginalized groups. It is hoped that the discussion presented in this paper may help to stimulate further debate on the relative applicability of intersectionality within the discipline of public health nursing and its potential to assist knowledge development in nursing. To sum up, intersectionality is a critical and fruitful theoretical framework that is sympathetic to the ontological and epistemological bases of the nursing discipline. Furthermore, it allows public health nurses to gain an awareness of the various forms of health disparities and inequities that are present among multiple historically oppressed and marginalized populations.

\section{CONFlicts OF INTEREST Disclosure}

The author declares no conflicts of interest.

\section{REFERENCES}

[1] Crenshaw K. Intersectional Feminism: Moving Toward a More Inclusive Practice. 2017; Available from: http://www.colorado.edu/studentaffairs/intersectional-feminism.

[2] Bilge S. Recent feminist outlooks on intersectionality. Diogenes. 2010; 57(1): 58-72. https ://doi.org/10.1177/039219211037 4245

[3] Bilge S. Intersectionality undone. Du Bois Review: Social Science Research on Race. 2013; 10(02): 405-424. https ://doi .org/10 $.1017 / \mathrm{S} 1742058 \mathrm{X} 13000283$

[4] Bowleg L. The problem with the phrase women and minorities: intersectionality - an important theoretical framework for public health. American Journal of Public Health. 2012; 102(7): 12671273. PMid:22594719 https://doi .org/10.2105/AJPH. 2012. 300750

[5] Crenshaw K. Demarginalizing the intersection of race and sex: A black feminist critique of antidiscrimination doctrine, feminist theory and antiracist politics. University of Chicago Legal Forum. 1989; 1989(1): 139-167.

[6] Guruge S, Khanlou N. Intersectionalities of influence: Researching the health of immigrant and refugee women. Canadian Journal of Nursing Research. 2004; 36(3): 32-47.

[7] Hankivsky O, et al. An intersectionality-based policy analysis framework: critical reflections on a methodology for advancing equity. International Journal for Equity in Health. 2014; 13(1): 119 PMid:25492385 https://doi .org/10.1186/s12939-014-011 $9-\mathrm{x}$

[8] Hankivsky O, et al. Exploring the promises of intersectionality for advancing women's health research. International Journal for Equity in Health. 2010; 9(1): 5. PMid:20181225 https ://doi .org/10.1 186/1475-9276-9-5
[9] Walby S, J. Armstrong, and S. Strid, Intersectionality: Multiple inequalities in social theory. Sociology. 2012; 46(2): 224-240. https ://doi .org/10.1177/0038038511416164

[10] Campbell ML. Intersectionality, Policy-Oriented Research and the Social Relations of Knowing. Gender, Work \& Organization. 2015; 23(3): 248-260. https : //doi.org/10.1111/gwao. 12083

[11] Davis K. Intersectionality as Buzzword: A Sociology of Science Perspective on What Makes a Feminist Theory Successful. Feminist Theory. 2008; 9(1): 67-85. https : //doi .org/10.1177/146470 0108086364

[12] Hakin R. Meeting the perspectives: Vulnerability and intersectionality for better health policies. in Paper presented at the International Conference on Public Policy. 2015. Milan.

[13] Kapilashrami A, Hill S, Meer N. What can health inequalities researchers learn from an intersectionality perspective? Understanding social dynamics with an inter-categorical approach? Social Theory \& Health. 2015; 13(3-4): 288-307. https : //doi.org/10.1057/st h. 2015.16

[14] Kagan PN, Smith MC, Chinn PL. Philosophies and practices of emancipatory nursing: Social justice as praxis. New York: Routledge. 2014.

[15] Kirkham SR, Browne AJ. Toward a critical theoretical interpretation of social justice discourses in nursing. ANS. Advances in Nursing Science. 2006; 29(4): 324. PMid:17135801 https://doi.org/10 .1097/00012272-200610000-00006

[16] McCall L. The Complexity of Intersectionality. Signs. 2005; 30(3): 1771-1800. https://doi.org/10.1086/426800

[17] Rodriguez JK, et al. The Theory and Praxis of Intersectionality in Work and Organisations: Where Do We Go From Here? Gender, Work \& Organization. 2016; 23(3): 201-222. https : //doi.org/ 10.1111/gwao. 12131 
[18] Carper BA. Fundamental patterns of knowing in nursing. Advances in Nursing Science. 1978; 1(1): 13-24. PMid:110216 https ://doi . org/10.1097/00012272-197810000-00004

[19] Marks LW. The emancipatory praxis of integral nursing: The impact of human caring theory guided practice upon nursing qua nursing in an American Nurses Credentialing Center Magnet redesignated healthcare system. ProQuest Dissertations and Thesis Database. 2013.

[20] McEwen M. Philosophy, science, and nursing. 3rd ed. Theoretical basis for nursing. New York: Lippincott Williams \& Wilkins. 2007.

[21] Watson J. Nursing: The philosophy and science of caring Revised ed. Caring in nursing classics: An essential resource. Colorado: University Press of Colorado. 2012; 243-264.

[22] Chinn PL, Kramer MK. Integrated theory \& knowledge development in nursing. 8th ed. St. Louis: Elsevier Health Sciences. 2013.

[23] Deckha M. Is culture taboo-feminism, intersectionality, and culture talk in law. Canadian Journal of Women \& the Law. 2004; 16(1): 14-53.

[24] Lui WY. Rethinking cultural marginality in the postmodern age: dialogical ambivalence of the multicultural self, in Department of Communication. University of Jyväskylä. 2009.

[25] Holtslander LF. Ways of knowing hope: Carper's fundamental patterns as a guide for hope research with bereaved palliative caregivers. Nursing Outlook. 2008; 56(1): 25-30. PMid:18237621 https://doi.org/10.1016/j.outlook.2007.08.001

[26] McBride A, Hebson G, Holgate J. Intersectionality: are we taking enough notice in the field of work and employment relations? Work, Employment and Society. 2015; 29(2): 331-341. https: //doi.org/10.1177/0950017014538337

[27] Dagkas S. Problematizing social justice in health pedagogy and youth sport: intersectionality of race, ethnicity, and class. Research Quarterly for Exercise and Sport. 2016; 87(3): 221-229. PMid:27463227 https://doi.org/10.1080/02701367.2016.1198672

[28] Reimer-Kirkham S. Nursing research on religion and spirituality through a social justice lens. Advances in Nursing Science. 2014; 37(3): 249-257. PMid:25102215 https ://doi.org/10.1097/AN S. 0000000000000036

[29] Van Herk KA, Smith D, Andrew C. Examining our privileges and oppressions: incorporating an intersectionality paradigm into nursing. Nursing Inquiry. 2011; 18(1): 29-39. PMid:21281393 https: //doi.org/10.1111/j.1440-1800.2011.00539.x
[30] Freire P. Pedagogy of the oppressed. 30th ed. New York: Bloomsbury Publishing. 2014.

[31] Bowleg L. The problem with the phrase women and minorities: intersectionality-an important theoretical framework for public health. American Journal of Public Health. 2012; 102(7): 1267-1273. PMid:22594719 https://doi.org/10.2105/AJPH. 2012.3007 50

[32] Lenette C, Boddy J. Visual ethnography and refugee women: nuanced understandings of lived experiences. Qualitative Research Journal. 2013; 13(1): 72-89. https://doi.org/10.1108/144398813113 14621

[33] Pui-Hing Wong J, et al. An exploratory study on the mental health of immigrants, refugees and non-status people living with HIV in Toronto. International Journal of Migration, Health and Social Care. 2013; 9(3): 122-134. https://doi.org/10.1108/IJMHSC-07-2 013-0026

[34] Yacob-Haliso O. Intersectionality and Durable Solutions for Refugee Women in Africa. Journal of Peacebuilding \& Development. 2016; 11(3): 53-67. https://doi.org/10.1080/15423166.2016.12 36698

[35] Jatau M. Living Between Two Cultures: A Reproductive Health Journey of African Refugee Women. Arizona State University. 2011

[36] Collins PH. Gender, black feminism, and black political economy. The Annals of the American Academy of Political and Social Science. 2000; 568(1): 41-53. https://doi.org/10.1177/0002716200 56800105

[37] Yuval-Davis N. Intersectionality and feminist politics. European Journal of Women's Studies. 2006; 13(3): 193-209. https ://doi.or $\mathrm{g} / 10.1177 / 1350506806065752$

[38] Erevelles N, Minear A. Unspeakable offenses: Untangling race and disability in discourses of intersectionality. Journal of Literary \& Cultural Disability Studies. 2010; 4(2): 127-145. https: //doi.org/10.3828/jlcds.2010.11

[39] Bauer GR. Incorporating Intersectionality Theory into Population Health Research Methodology: Challenges and the Potential to Advance Health Equity. Social Science \& Medicine. 2014; 110: 10-17. PMid:24704889 https://doi .org/10.1016/j . socscimed. 20 14.03 .022

[40] Cho S, Crenshaw KW, McCall L. Toward a field of intersectionality studies: Theory, applications, and praxis. Signs. 2013; 38(4): 785-810. https://doi.org/10.1086/669608 